\title{
EDITORIAL
}

\section{A TEACHING GERIATRIC HOSPITAL}

\author{
F. MORALES-MARTÍNEZ
}

\begin{abstract}
General Director, National Geriatrics and Gerontology Hospital, San Jose, Costa Rica. Corresponding author: Dr. Fernando Morales-Martínez MD., AGSF, FRCP (Edin), General Director, Geriatrician and Gerontologist, National Geriatrics and Gerontology Hospital, Professor of Medicine University of Costa Rica San Jose, Costa Rica,

Email: fernandomm@costarricense.cr
\end{abstract}

This editorial outlines the importance of a teaching geriatric hospital in Costa Rica, the National Geriatrics and Gerontology Hospital (NGGH), and describes it's history, passing through different steps to become the specialized hospital for people 60 years and over and to reach important achievements as the national reference of elderly and ageing matters for the country. Since its creation, the hospital has had a great role in the community by joining in the efforts by establishing home visiting and domiciliary hospitalization as well as palliative care. It has liaisons with other nongovernmental organizations and the older person care network. Through the years, the NGGH has strengthened its teaching and research program, with the University of Costa Rica that stands out in quality. The NGGH is unique in Latin America because, as it is an acute geriatric hospital, it has all the services that meet the required assessment of the older person.

Costa Rica has a highly complex social security system that enables guaranteed access to medical assistance and assurance of revenues for disease and maternity issues, disability, and possible retirement funds at the end of their working career that goes into old age. The Costa Rican health system is universal, covering $96 \%$ of the total country's population for disability, age, death and health insurance for over 77 years through the Costa Rican Social Security Institute.

As part of this framework, the direct health attention that the National Geriatrics and Gerontology Hospital (NGGH) implements, stands out.

Adverse events in the hospitalized elderly have been described for many years and there have been many studies of the causes and consequences of hospital associated adverse events. In recent years however, research has focused on the preventable aspects of hospital adverse events in the elderly. In Europe, the United States, Australia and parts of East Asia studies have tested whether various modifications of usual hospital processes of care and environmental design can improve outcomes. Results of these studies have been extensively reviewed. The concept of an ACE unit, a geographically located hospital nursing unit for acute care of the elderly has gotten consistent research support. Similarly, the idea of transferring principles of care for the acutely ill elderly have been applied to dispersed ACE consultation teams. A multidisciplinary geriatric consult team directs the care of high risk patients on general inpatient services. These are codified in the widely disseminated HELP program. Both models of care have demonstrated decreased rates of incident delirium and improved discharge planning for elderly medical and surgical patients, including those in intensive care. Specifically, orthogeriatric care of hip fracture patients results in improved functional status and shorter hospital stays. In Europe, notably in the Netherlands and Great Britain, and Asia, there are medical care facilities called Geriatric Hospitals but they are principally providers of subacute and long-term care.

This leaves us to wonder why the idea of a dedicated ACE unit or even an entire hospital is still so neglected. The reasons are many, including competition for bed space in existing hospitals, emphasis on income generating procedures, buy-in from administrators and shortages of trained geriatric providers. However, the idea of an entire hospital for acute care of the elderly has its advocates. The present report describes the unique history and development of a free-standing geriatric hospital in Costa Rica, the National Geriatrics and Gerontology Hospital (NGGH). We describe the social, population and health system priorities that supported its development and plans for the future.

\section{History}

In 1975 the National Tuberculosis Hospital in San Jose, which was built in 1958 was renamed for eminent tuberculosis specialist Dr. Raul Blanco Cervantes and then closed due to changes in the clinical management of tuberculosis and transfer of patients to outpatient care. The building was transferred the next year to the Costa Rican Social Security Institute. In 1989 legislation entitled the National Health System Regulation which supported the development of national centers (National Specialized Hospitals). With this designation the NGGH became a teaching and research center for geriatrics and gerontology. In 1992 this mission was formalized by a collaborative agreement with CCSS, and the residency programs of the School of Medicine of the University of Costa Rica.

In 1999 legislation was passed to assure that elderly would have equal access to hospital, emergency, outpatient, rehabilitative and preventive care. This law mandated that geriatric specialty services be established in each state general hospitals, regional hospitals and referral outpatient clinics. The law provided for resources to staff and operate the services and specified that they be advised by the Dr. Raul Blanco Cervantes NGGH in San Jose. Currently there are 134 geriatricians working in the Costa Rican health system and 60 geriatric MD 


\section{THE JOURNAL OF NUTRITION, HEALTH \& AGING@}

trainees. Presently there is about 1 geriatrician per 4200 elderly in Costa Rica. Two-thirds of them are practicing in the capital province of San Jose (Figure 1).

Figure 1

Distribution of geriatricians in the different hospitals of the CCSS, 2018

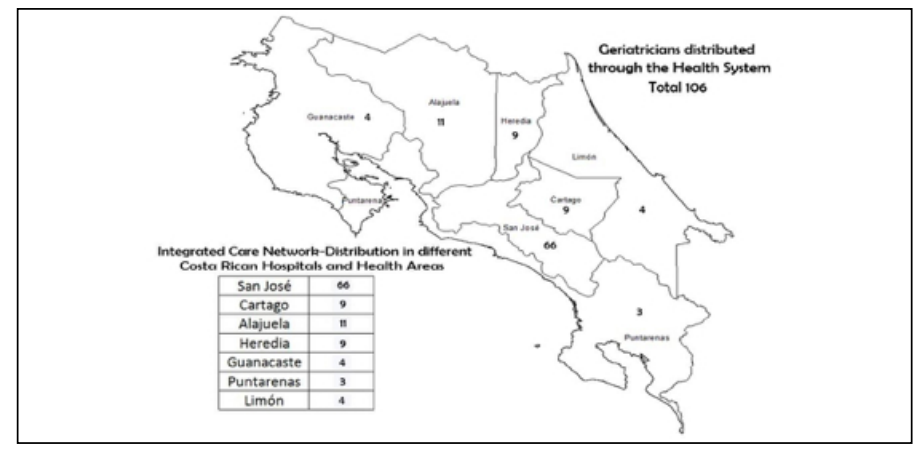

Source: Geriatric and Gerontology Clinical Department. National Geriatrics and Gerontology Hospital, 2018

The research and educational mission of the NGGH continues to expand. It is a designated Collaborative Center of the World Health Organization. Since 2008 more 114 health care professionals from 14 countries have completed fellowship training at NGGH. In 2016 the framework for the future of comprehensive, integrated care for the elderly was proposed to the Social Security Administration of Costa Rica as the Project for a Tower of Integrated and Integral Geriatric Services. This proposal requests a new hospital building with expanded surgical and emergency capacity to serve the network of 29 referral hospitals around the country.

\section{Population Imperative}

Costa Rica is a middle-income country. It's vital statistics including maternal and child health compare favorably with EU and the USA. The fertility rate is also declining. In 2013, the fertility rate dropped to 17.6 live births per 1,000 women of childbearing age which is below the replacement rate of 21.0 per 1,000 that is generally used to anticipate population growth or decline. The squaring of the population pyramid is seen in the decline of those less than 15 years from $31.9 \%$ in 200 to $24.8 \%$ in 2011. Concurrently, the proportion of those aged 65 and older increased 5.3\% in 2000 to $7.7 \%$ in 2018. In 1950 median life expectancy was $\mathbf{5 7 . 3}$ years, today it is 79.8 years. Projections to 2050 are predicting a median life expectancy of 82 years. With birth rates declining below replacement rates and population aging, national policies have favored preparation for future health care needs.

\section{Referrals and Discharges}

The social security system of Costa Rica has been in place for 77 years. It provides essentially universal (96\%), health care including maternity care, disability and retirement income subsidies. With universal health care coverage, there are 102 public health districts served by 29 hospitals. 22 of these have staff geriatricians who have no insurance barriers to referral to the national geriatric hospital, NGGH. Referrals come from any of the national network of hospitals and clinics. Adults aged 60 and older requiring specialized geriatric care can be referred to the central NGGH. We treated 57593 patients in the out-patient department last year, 2792 patients of the in-patient department and 19460 patients from the emergency department with a mean age of 84 years. The mean stay was 16.2 days. Patients are triaged to acute 137 beds) and intensive 134 beds) care or directly to subacute care for rehabilitation from strokes, fractures and other immobilizing conditions. More patients not requiring admission or no longer requiring inpatient care can be treated at the Day Hospital, outpatient consultation clinics or community care. When patients are ready to return home, NGGH staff coordinates with the community providers to whom the patient will return.

\section{What are the components of the NGGH care continuum?}

As we have described, the geriatric acute care hospital functions as an important node in an integrated network of services and centers along a continuum of acuity. Much of our success is attributable to the investment in care coordination among the components of our geriatrics center on campus, locally and with health centers throughout the country. At each transition, admission and discharge, the interdisciplinary team meets with families and patients to lay out next steps and explain the patients' needs.

The heart of NGGH campus is the acute care hospital which houses the Emergency Department, all acute inpatient medical and surgical patients, the operating rooms, and the support services including radiology, laboratories, and pharmacy. The acute hospital provides comprehensive diagnostic and treatment services for referral patients with a 30-day limit. There is an average 8-day length of stay for acute care patients. The subacute rehabilitation unit located in the hospital building provides for up to 90 -day stays.

Since 2009, the ambulatory program has been housed in its own building, the Integrated Ambulatory Geriatric Services (IAGS) building. Outpatient geriatric consultation, dentistry, the Day Hospital, Outpatient Pharmacy, the domiciliary Geriatric Community Unit (GCU) which also provides palliative care, and the offices of the academic and clinical departments of Geriatrics and Gerontology of the University of Costa Rica. The expanded outpatient clinics have provided a home for a number of specialty programs in addition to the usual subspecialties such as endocrinology, cardiology, otolaryngology and so forth. The IAGS houses CIENAAA, an interdisciplinary research and clinical program dedicated to elder abuse and neglect. The memory clinic specializes in studies of Mild Cognitive Impairment (MCI.) There is a clinic to study sleep disorders. 


\section{A TEACHING GERIATRIC HOSPITAL}

The Nutritional Support clinic accepts referrals for malnutrition. There are also specialty clinics for sarcopenic obesity, bereavement, pressure ulcers, geriatric oncology, falls and pain management. As a branch of the NGGH-IAGS continuum, the center has established home visiting health care and palliative care services.

\section{Implications for practice}

The NGGH is a center for interdisciplinary care and research for the growing geriatric population of Costa Rica and it is a recognized resource for geriatric research and clinical care throughout Latin America. We began with our unique acute geriatric hospital. It became the foundation of a differentiated continuum care network. At the core of the system is training the multidisciplinary professionals who are essential to managing the transitions along the continuum of care and to articulating the NGGH values for care of the aged throughout the health care system.

Costa Rica has shown rapid growth of the elderly population. The life expectancy in 1950 was 57.3 years, while today it is 79.8 years. It is expected that by 2050 life expectancy will reach 82 years; this places Costa Rica in 34th place worldwide and 1st place in Latin America. The annual population growth rate is $1.4 \%$ due to a moderate birth rate (18 per 1000) and a low mortality rate $(4.3$ per 1000$)$. In 2017 , the Supreme Electoral Tribunal reported 680 centenarians. Three quarters of them were male and one quarter female. The information from the National Institute of Statistics and Census (INEC) has shown a decrease in birth rates (from 2.12 children per females to 1.76 in 2015). This is clear evidence that the Costa Rican populace is in a continuous process of demographic ageing and Costa Rica is now below the maintenance replace rate of 2.16 per female.

The population less than 15 years of age is decreasing from $31.9 \%$ in 2000 to $24.8 \%$ in 2011 ; while the 65 years and older population is increasing from $5.3 \%$ in 2000 to $7.7 \%$ in 2018 (Figure 2).

\section{Figure 2}

Demographic situation: Projections of the population structure from 2000 to 2100, Costa Rica

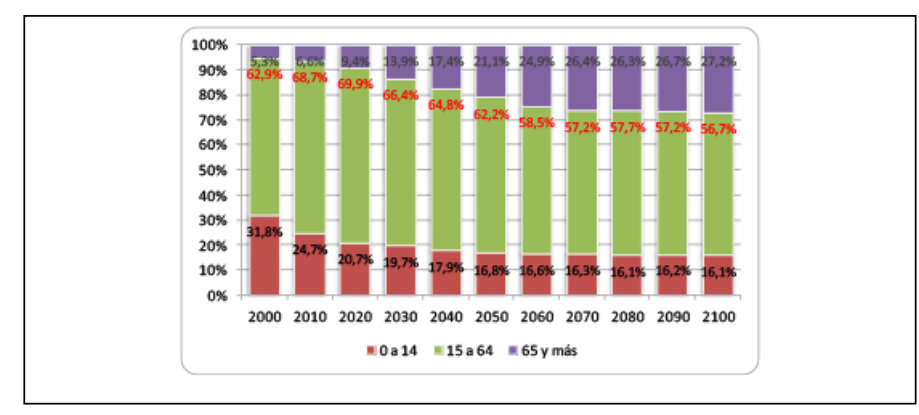

Source: Area of Health Statistics, CCSS
At its inauguration in January of 1958 through 1975, the building that now is the National Geriatrics and Gerontology Hospital was known as the National Tuberculosis Hospital. The main objective was to provide direct attention to the population that were diagnosed with a disease that required long hospital stays during that period. As of December 1975, the hospital was named after Dr. Raul Blanco Cervantes (in honor to the Tuberculosis specialist). At the time, important advances in medicine favored treating persons with Tuberculosis in the community-dwelling scenario and within their families. This major change triggered a transition process of the center, to a direct intervention for the health of the elderly.

In 1976, the hospital was transferred to the Costa Rican Social Security Institute (CCSS) who decided that health attention of the elderly was a major priority. This lead to the development of specialized attention to geriatrics and gerontology. In 1989, the National Health System Regulation was enacted which included a reframing of the health centers activities. In this same decree, the categorization of the National Specialized Hospitals was defined, where all would belong to the level III of attention. This included the National Geriatrics and Gerontology Hospital. Thus, it became a teaching and research center for the care of older persons.

This approach was in accordance with the Integral Law of the Elderly Person \#7935 (1999) article 3, which stated that all elderly have the right to immediate hospital, emergency, preventive, clinical and rehabilitation attention. At that time each state was responsible to create geriatric services in all national general hospitals as well as in regional hospitals and level III and IV out-patient clinics. These medical centers should have specialized personnel in the field, with adequate physical, human and financial resources to guarantee efficient attention to the patient and must be advised by the National Geriatric and Gerontology Hospital Dr. Raul Blanco Cervantes.

The development of the National Geriatrics and Gerontology Hospital is seen as a university hospital due to an agreement between the CCSS and post graduate program of the School of Medicine of the University of Costa Rica in 1992. There are 134 specialists in Geriatrics and Gerontology in Costa Rica with a distribution rate of roughly 4200 older persons per geriatrician. A total of 106 specialists work for the Costa Rican health system. Figure 1 gives the distribution of these geriatricians within the provinces of the country. In addition, there are 60 in-training geriatricians in the five-year program at the National Geriatric and Gerontology Hospital.

Of the 29 hospitals and 102 health areas in Costa Rica, 22 centers have geriatricians and gerontologists. The referral process to the National Geriatrics and Gerontology Hospital is done according to the complexity of geriatric cases. Specifically, at the NGGH, only people of 60 years of age and over are admitted. The mean age of attendance is 84 years. The elderly may be evaluated at the Diagnostics and Treatment Service, for the study and integral assessment. Once a course of action is defined, he or she can be admitted to the Functional 


\section{THE JOURNAL OF NUTRITION, HEALTH \& AGING@}

Recovery Unit to receive treatment for stroke sequels, fractures and/or immobilization syndromes. Acute cases are treated in the Immediate Care Unit (myocardial infarction, acute respiratory distress, and diabetic comas). If the case is considered for ambulatory care, it is referred to the Day Hospital, Out-patient Consultation or the Geriatric Community Care Unit.

Once the elderly person has received the specialized attention, the health team plans his or her discharge with appropriate medical treatment and integral follow-up at the health area, nursing home, Day Care Center, Sheltered housing, or family domiciliary scenario within the community. A summary of the integral and dynamic health attention that is given through close relationship with the family and community, taking maximum advantage of the available resources, is described in Figure 3.

\section{Figure 3}

Progressive attention network for the elderly, Costa Rica

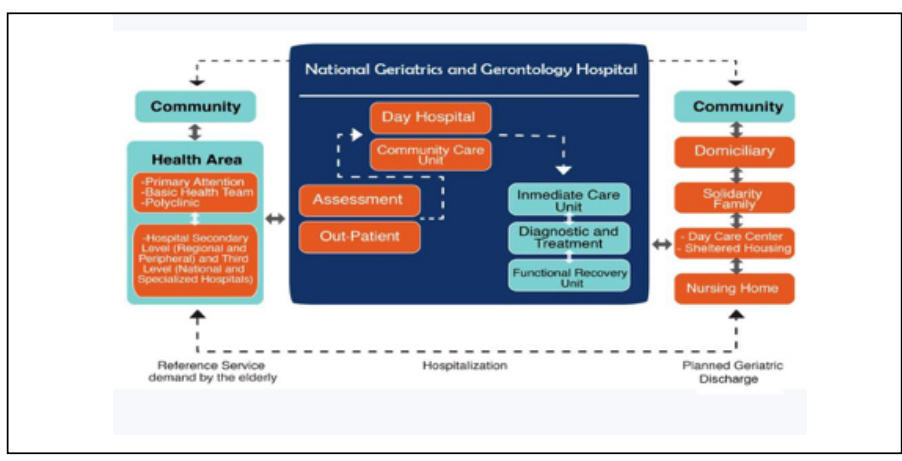

Source: Geriatric and Gerontology Clinic Department, National Geriatrics and Gerontology Hospital, 2018

The geriatric hospital currently consists of:

1. The main building where the Emergency Service, hospitalization, surgery, procedures and all evaluation and interdisciplinary services are located, include all different support services (X-Rays, Pharmacy and Laboratory, among others); and

2. The Integral Ambulatory Geriatric Service building (SIGA), which began operations in 2009, harbor their programs: Out-patient Consultation, Odontology, Day Hospital, Pharmacy, Geriatric Community Unit (domiciliary and palliative care) Academic Department and the Clinic Department of Geriatrics and Gerontology of the University of Costa Rica.

Many specific clinics have been developed:

- Integral Study of the Abused, Assaulted and Abandoned Elderly Committee (CEINAAA), exists since 1996 and is dedicated to the interdisciplinary and integral cases of mistreated elderly identified in the different health attention services.

- A memory clinic to study mild memory impairment.

- Sleep disorders clinic for cases that present difficulties with medical treatment.

- Nutritional Support clinic to pay attention to cases with malnutrion

- A clinic for sarcopenia obesity.

- Bereavement clinic

- Pressure ulcers clinic

- Oncology clinic

- Falls, with a focus on causes and their treatment.

- Pain clinic.

In the hospital there are:

- Diagnostic and Treatment care for patients who are studied and have hospital stays of up to 30 days maximum

- A functional Recovery unit for hospital stays up to 90 days maximum.

- Immediate Care, acute cases that have hospital stays of up to 8 days.

- Surgical service

On admission and discharge a team meeting is carried out with the patient, and their families, with nursing personnel to allow understanding of the process and needs of the patient.

On December 2016, the Project of the Tower of Integrated and Integral Geriatric Service was presented to the authorities of the CCSS in which the necessity of an extension of the infrastructure of the NGGH was outlined. In the project the proposal of a new building that will harbor a 24/7 Emergency Service, extend the number of hospital beds, extend surgery rooms and surgical specialties including Orthopedics, Otorhinolaryngology and Oncology which currently are not available. The project also includes the extension of already available services such as: Ophthalmology, General Surgery, Urology as well as Dermatology and Odontology along with improvement of support services of the Laboratory, X-Rays and Pharmacy.

The main tasks of the National Geriatrics and Gerontology Hospital, as a center that brings highly complex specialized health attentions for the elderly across the country, referred by the different health areas, including the formation of specialists in Geriatrics and Gerontology that are integrated within the health services network composed of 29 hospitals are acknowledged. The hospital is head of a dynamic national geriatric leadership that requires the strengthening of coordination efforts at the interinstitutional level with all public and private organizations, dedicated to providing different services for the elderly. It is necessary to development actions to prepare professional and administrative health personnel along with investigation in the field of geriatrics and gerontology to articulate specialized services to diagnose and treat, for and develop norms for permanent actualization at national level. 


\section{Implications for the community and for the world}

The current proposal of coordinating, in a more efficient manner, the national geriatric network by strengthening the relationship: hospital-community-family, the civil society, community organization, volunteering and the older person care network, for university teaching and investigation of prevalent diseases in the country must be integrated in all national policies. There is a written commitment by the in-coming administration (2018-2022) to create this new hospital in the very near future.

Currently, the NGGH is a Collaborative Center of the World Health Organization, unique in Latin America. Since 2008, more than 110 professionals from 14 countries have done a fellowship in the hospital. To date, the NGGH continues to be much active in specialized services, teaching and investigation. To our knowledge this is the only acute service Geriatric Hospital in the world. Geriatric hospitals in the rest of the world tend to be subacute or rehabilitation hospitals. Our hospital is a trail blazer following the pathway of the development of pediatric hospitals in the 20th century.
Acknowledgements: The author wishes to acknowledge MSc. Yolanda BrenesZumbado and Dr. Manrique Sandi-Arias for their support in revising this paper.

Conflict of interests: The Editor-in-Chief has reviewed the conflict of interest checklist provided by the author and has determined that the author has no financial or any other kind of personal conflicts in the preparation of this paper.

Author contributions: Fernando Morales-Martinez was the sole author of this article.

Sponsor's role: None

\section{References}

1. CEPAL. Economic Commission for Latin America. Demographic transformations and its influence in the development of Latin American and the Caribbean. 32 nd Document, CEPAL, period of sessions. Santo Domingo pp 145, 2008

2. Martínez Franzoni, Juliana. The social security in Costa Rica: Perceptions and experiences of those who have less and need it more. Interamerican Bank for Development; Department of Sustainable Development, Washington, D.C pp 38, 2006

3. FESS Program. Building a strategic agenda for the strengthening of the equity and the equity and sustainability of the health insurance. San Jose, 2014

4. Solis Guevara, Ana. Use of Health Services for the Elderly. Area of Health Statistic, CCSS, report of the social security system. San Jose, 2013

5. Morales Martínez, Fernando. Is Geriatric Medicine Possible in Midle-Income Country? The Case of Costa Rica. In: Journal of the American Geriatrics Society, JACS. 2017;August. Volume 65. Issue 8. pp 1870 - 1875.

6. Morales Martínez, Fernando. Geriatric medicine bridges: Scotland - Costa Rica. In: Journal of the Royal College Physicians of Edin JR Coll Physicians Edinburgh. December. 2017; Volume 47. Issue 4. pp 324-330 